Ciencia Animal y Pastilzales/Animal Science and Grasslands

Cienc Tecn UTEQ (2018) 11(2) p 9- 15 ISSN 1390-4051; e-ISSN 1390-4043 (c) (i) (8) (9) DOI: https://doi.org/10.18779/cyt.v11i2.260

\title{
Degradabilidad ruminal del rastrojo de arroz tratado con urea en rumiantes
}

\section{Rumen degradability of urea treatment rice straw in ruminants}

Torres Ayala María Belén¹, Hernández Silva Jaime Marcelo¹, Grijalva Olmedo Jorge Eduardo²

\author{
${ }^{l}$ Ministerio de Agricultura, Ganadería, Acuacultura y Pesca, Central Nacional de Producción y Mejoramiento Genético El Rosario, Miraflores \\ Alto, Tambillo, Ecuador \\ ${ }^{2}$ Universidad Central de Ecuador, Facultad de Medicina Veterinaria y Zootecnia, Jerónimo Leyton y Gato Sobral, Quito, Ecuador. \\ Telef.: +59322548523, Email: jgrijalva@uce.edu.ec
}

\begin{abstract}
Resumen
$\mathrm{E}$ rastrojo de arroz constituido por tallos y hojas, puede ser un importante subproducto de cosecha del grano de arroz para ser utilizado en la dieta de rumiantes, en razón de su abundante disponibilidad. El aumento de las regulaciones y restricciones sobre la quema de rastrojos, ha estimulado el interés por usar para otros propósitos, incluyendo la alimentación del ganado. La técnica de degradación in situ ha sido ampliamente adoptada para evaluar la tasa de degradación de alimentos en el rumen. El objetivo del trabajo fue evaluar mediante la técnica de degradación ruminal in situ, la degradabilidad de la materia seca (MS) del rastrojo de arroz tratado con seis niveles de urea: 0, 5, 10, 20, 30 y $40 \mathrm{~g} \mathrm{~kg} \mathrm{MS}^{-1}$. Los sustratos secos fueron fragmentados en dos tamaños de partícula: una parte picada a 2 $\mathrm{cm}$ y otra parte molida a $2 \mathrm{~mm}$, ambos tipos de sustratos fueron incubados en horno de aire forzado a $40{ }^{\circ} \mathrm{C}$ durante 10 días. Se utilizaron ocho vacas provistas de cánula ruminal para los ensayos de digestibilidad in situ, a 48 y 96 horas de incubación en rumen. Cuatro vacas recibieron una Dieta de Alta Actividad Ruminal (AAR) ajustada a consumo de $5 \mathrm{~kg} \mathrm{MS} \mathrm{día}{ }^{-1}$ de heno de alfalfa (Medicago sativa L.) + heno de una mezcla forrajera (Pennisetum clandestinum Hochst. ex Chiov, Lolium perenne L. y Dactilys glomerata L.) ad libitum $+1.8 \mathrm{~kg}$ de concentrado, y cuatro vacas restantes recibieron una Dieta de Baja Actividad Ruminal (BAR) basada en heno de la misma mezcla forrajera y concentrado. Los datos fueron analizados mediante regresión múltiple por pasos sucesivos. Los resultados muestran aumentos en la degradabilidad de la MS con el incremento en el nivel de urea en dietas con AAR y a 96 horas de incubación. El tamaño de partícula no afectó la respuesta animal. Se concluye que este subproducto de cosecha tratado con urea puede ser una importante fuente de fibra para rumiantes en los trópicos.
\end{abstract}

Palabras clave: Degradación ruminal, rastrojo de arroz, tratamiento con urea.
Rec.: 05.11.2018. Acept.: 17.12.2018 Publicado el 30 de diciembre de 2018

\begin{abstract}
$\mathbf{R}$ ice straw, both its leaves and stems, could be an important product of the harvest of rice to be used in animal nutrition because of its high availability. Increasing regulations and restrictions on the practice of burn rice straw have stimulated the exploration of other purposes for such straw, including animal feeding. The in-situ method has been widely used in the evaluation of the ruminal degradability rate of several animal feeds. The objective of this study was to evaluate the effect of urea addition on the dry matter (DM) degradability of rice straw. Six levels of urea addition were tested: $0,5,10,20,30$ and $40 \mathrm{~g} \mathrm{~kg}$ $\mathrm{DM}^{-1}$. The rice straw was chopped to $2.0 \mathrm{~cm}$ long pieces or grinded to $2.0 \mathrm{~mm}$ particles and incubated in presence of urea for 10 days at a moisture content of $66 \%$ at a temperature of $40{ }^{\circ} \mathrm{C}$ to simulate optimum environmental conditions. Eight fistulated Holstein Friesian cows were used to study the Dry Matter Digestibility at 48 and 96 hours, and were separated into two groups: one fed with a High Ruminal Activity (AAR) diet based on $5 \mathrm{~kg}$ of lucerne (Medicago sativa $\mathrm{L}$ ) hay, ad libitum forage mixture (made of Pennisetum clandestinum Hochst. ex Chiov, Lolium perenne L and Dactilys glomerata L) and $1.8 \mathrm{~kg}$ of concentrate, per day. While the other group received a Low Ruminal Activity (BAR) diet composed only of the ad libitum forage mix and the concentrate. Degradability data was analyzed using a Stepwise Multiple Regression. The results show that degradability of DM increases with higher levels of urea addition, the same as with the AAR diet and with higher ruminal incubation time. Different particle sizes didn't show any difference. The conclusion is that this straw could be an important fiber source for ruminants if treated with urea, especially in the tropical region.
\end{abstract}

Keywords: Rice straw, rumen degradation, urea treatment. 


\section{Introducción}

$\mathrm{U}$ no de los actuales desafíos que enfrenta la actividad ganadera en América Latina y el Caribe, es la creciente demanda de productos como la leche, carne y derivados de alta calidad, lo cual exige que la nutrición de los animales sea cada vez más eficiente y rentable (Garg, 2012). De hecho, más del $70 \%$ de los costos de producción de leche o de carne, se atribuyen a la alimentación.

En esta región, se reconoce la gran variabilidad en la disponibilidad y calidad de los recursos forrajeros tropicales, generalmente los elementos nutritivos son bajos e insuficientes para una adecuada nutrición de rumiantes, hecho que limita significativamente la producción bovina (Rosero y Posada, 2007). Al respecto, se sostiene que el valor real de un alimento para el animal, solamente puede ser determinado tomando en cuenta los efectos durante el proceso de digestión (Pond et al., 2006), y para ello, se requiere de pruebas de digestibilidad in vivo, in situ y/o in vitro (Moran, 2005; Posada y Noguera, 2005). La digestibilidad por el método in situ o de bolsas nylon, es parte de estas últimas técnicas, también denominadas de digestibilidad enzimática, producción de gases y de las bolsas suspendidas en rumen, y consiste en la incubación de muestras en estudio en fundas nylon en el rumen de bovinos fistulados (Orskov and Shands, 1997; Pulido y Leaver, 2000; Villalobos et al., 2000; Arreaza et al., 2005; Rossi et al., 2008; Torres et al., 2009). Todas estas técnicas permiten estimar la digestibilidad y la dinámica de degradación ruminal de las fracciones nutritivas de los alimentos, con el fin de estudiar las variaciones en la respuesta animal ante cambios en las condiciones ruminales (France et al., 2000).

El sistema de producción dominante de la ganadería ecuatoriana es el pastoreo directo, más el uso de niveles variables de suplementación con alimentos concentrados basados en insumos de alta concentración energética y moderadas cantidades de subproductos y residuos provenientes de la actividad agrícola. El residuo de la cosecha de arroz (Oriza sativa), conocido como paja o rastrojo de arroz podría destinarse a la nutrición animal, tal como se realiza en otras regiones del mundo de alta producción de este grano (Sarnklong et al., 2010). La superficie aproximada de cultivo de arroz en el país durante 2014 fue de 375 mil hectáreas con un rendimiento de 1.4 millones de toneladas anuales (INEC-ESPAC, 2014), lo cual supone que esta actividad genera importantes cantidades de rastrojos poscosecha que se quedan en el terreno como fuente de materia orgánica, a pesar de que podría ser utilizado en la nutrición animal (Haryanto, 2014).

El principal obstáculo para la utilización masiva del rastrojo de arroz en la ganadería, es su bajo valor nutritivo y escasa palatabilidad (Drake et al., 2002; Bartaburo et al., 2008), por lo que se recomienda su uso como reemplazo parcial de la ración diaria y no como fuente principal de alimento (Malik et al., 2015). Estudios previos han demostrado que la composición química de la paja de arroz es muy variable, conteniendo 3-5\% de proteína cruda (PC) (Drake et al., 2002; INTA, 2014), 45\% de digestibilidad aparente de la materia seca (Van Soest, 2006), 50\% de FDN y alrededor de 1.84 Mcal de EM (Manterola y Cerda, 2017). Adicionalmente, acusa un alto contenido de sílice que limita el consumo voluntario en rumiantes (Drake et al., 2002). Es importante mencionar que la mayor cantidad de sílice se concentra en la hoja del rastrojo y no en el tallo, razón por lo cual esta última fracción sería comparativamente más digerible (Jackson, 1997; Van Soest, 2006).

Se han desarrollado varios métodos para mejorar la calidad nutricional y digestibilidad del rastrojo de arroz, que incluyen la aplicación de tratamientos químicos, físicos y microbiológicos de mayor o menor complejidad (Liu et al., 1999; Arreaza et al., 2005; Torres et al., 2009; Bartaburu et al., 2008; Sarnklong et al., 2010). Entre los métodos químicos, la aplicación de urea es uno de los más extendidos por su facilidad de utilizar y costo relativamente bajo (Vadiveloo y Fadel, 2009), además, la urea aporta nitrógeno extra a la dieta lo cual estimula la actividad ruminal (Malik et al., 2015).

Numerosas investigaciones han afirmado que el tratamiento del rastrojo de arroz con urea es una medida eficaz para mejorar la calidad nutricional (Yulistiani et al., 2008; Vadiveloo y Fadel, 2009; Wanapat et al., 2009; Gunun et al., 2013), pero aún parecen persistir dudas sobre las múltiples causas que expliquen satisfactoriamente ese hecho. Por lo expuesto, el propósito de este estudio consistió en evaluar el efecto de la adición de urea al rastrojo de arroz sobre la degradabilidad ruminal o "in situ" de la materia seca, y examinar si otros factores tales como la calidad de dieta, el tamaño de partícula y el tiempo de permanencia de partículas en el rumen, son factores que afectan la digestibilidad de este subproducto.

\section{Materiales y métodos}

\section{Recolección y homogenización de muestras de rastrojo de arroz}

Se utilizó el rastrojo de la variedad de arroz INIAP 14, proveniente de plantas cosechadas a los 120 días desde la siembra. De un total de 100 fardos recolectados en la parroquia Daule de la provincia del Guayas, se tomó una muestra compuesta de 20 fardos de 8,5 kg de peso cada uno, los cuales fueron cuidadosamente mezclados hasta obtener 120 sub-muestras homogéneas con pesos aproximados de 1,4 $\mathrm{kg}$. El 50\% de estas sub-muestras se fraccionaron o picaron a $2 \mathrm{~cm}$ de longitud de partícula y el $50 \%$ restante se molió a 2 $\mathrm{mm}$ de tamaño de partícula. Las muestras fueron secadas a 60 ${ }^{\circ} \mathrm{C}$ durante 24 horas hasta obtener peso constante.

\section{Preparación y administración de dietas experimentales} Se utilizaron ocho vacas Holstein Friesian provistas 
de una cánula ruminal, de 3.8 años de edad y $580 \mathrm{~kg}$ de peso vivo, cuatro de las cuales recibieron una ración de alta actividad ruminal (AAR) siguiendo procedimientos sugeridos por varios autores (Yulistiani et al., 2015; Giraldo et al., 2007). Esta dieta estaba conformada por heno de $M$. sativa (170 $\mathrm{g}$ de $\left.\mathrm{PC} \mathrm{kg} \mathrm{MS}^{-1}\right)$ calculada en orden a permitir un consumo de $5 \mathrm{~kg} \mathrm{MS} \mathrm{vaca-1}$ día $^{-1}+$ consumo ad libitum de heno de una mezcla forrajera a base de Lolium perenne, Pennisetum clandestinum y Dactylis glomerata $(80 \mathrm{~g} \mathrm{PC} \mathrm{kg}$ $\left.\mathrm{MS}^{-1}\right)+1.8 \mathrm{~kg}$ de un concentrado comercial (160 g PC y 3.0 Mcal EM kg MS ${ }^{-1}$ ). Las restantes cuatro vacas recibieron una dieta de baja actividad ruminal (BAR) conformada únicamente por heno de la mezcla forrajera ofrecida ad libitum y la misma cantidad de concentrado que la dieta AAR. Las unidades experimentales fueron confinadas por separado y alimentadas con una de las dietas durante catorce días de adaptación con el propósito de acostumbrar al rumen a las características de cada régimen, tal como recomiendan estudios previos (Saddul et al., 2005). El cuidado y manejo de las vacas fistuladas se realizó en base a la guía establecida por la Federation of Animal Science Societies (FASS, 2010).

\section{Tratamiento del rastrojo de arroz con urea}

La adición de urea en el rastrojo de arroz se basó en el método utilizado por Yulistiani et al. (2008). Para el efecto, se preparó aproximadamente $2000 \mathrm{~g}$ de paja de arroz molida a $2 \mathrm{~mm}$ y $2000 \mathrm{~g}$ de paja de arroz picada a $2 \mathrm{~cm}$ de tamaño de partícula. Se evaluaron seis niveles de urea. El nivel 0 correspondió al control sin adición de urea. La aplicación de urea se hizo utilizando un aspersor y mediante volteo continuo del material, se obtuvieron muestras homogéneas. Los niveles de urea aplicados se expresan en el Cuadro 1.

El contenido de $\mathrm{N}$ del rastrojo N0 sin urea, correspondió a un $7.8 \%$ de "proteína cruda equivalente". Posterior a la adición de la solución de urea, se adicionó agua hasta que las muestras alcanzaron un $66.6 \%$ de humedad. Las muestras fueron finalmente colocadas en frascos de plástico tapados y sellados, y se incubaron a $40{ }^{\circ} \mathrm{C}$ durante 10 días, simulando óptimas condiciones.

Cuadro 1. Nivel de urea aplicado y contenido de $\mathbf{N}$ en el rastrojo de arroz

\begin{tabular}{ccc}
\hline $\begin{array}{c}\text { Nivel urea } \\
{[\mathbf{N}]}\end{array}$ & $\begin{array}{c}\text { Cantidad urea } \\
\text { [g kg-1 de rastrojo] }\end{array}$ & $\begin{array}{c}\text { Nitrógeno en } \\
\text { rastrojo [\%] }\end{array}$ \\
\hline N0 & 0 & 1.26 \\
N1 & 5 & 1.49 \\
N2 & 10 & 1.72 \\
N3 & 20 & 2.18 \\
N4 & 30 & 2.64 \\
N5 & 40 & 3.10 \\
\hline
\end{tabular}

Determinación de la Degradabilidad ruminal de la Materia Seca (DMS) del rastrojo de arroz

La determinación de DMS se basó en el método in situ utilizado por Ørskov y Shand (1997). Para el efecto, diez gramos de muestras secas de la paja de arroz molida y picada, se colocaron en bolsas de nylon de 10x20 cm, semipermeables con poros de 53 micrones de diámetro, para permitir el contacto del líquido ruminal con el sustrato. Estas bolsas fueron adecuadamente selladas y posteriormente colocadas en el rumen y sujetas con hilos a la tapa de la fístula durante 48 y 96 horas. Al respecto, se ha demostrado que el tiempo de residencia de los alimentos en el rumen generalmente es de 48 horas (Ørskov y Shand, 1997). Al finalizar el tiempo de incubación en rumen, las bolsas fueron extraídas y lavadas con agua hasta retirar aparentemente todos los residuos de contenido ruminal, es decir cuando el agua de lavado dejó de presentar turbidez. Finalmente, las muestras se secaron a 60 ${ }^{\circ} \mathrm{C}$ por 48 horas en horno de aire forzado luego de lo cual se pesaron.

La DMS se definió como el porcentaje de materia seca de la muestra perdida durante la incubación en rumen, cuyo valor fue determinado mediante la diferencia de peso de las bolsas antes y después de su incubación en el rumen, como se muestra en la Ecuación 1:

DMS $=\frac{\text { Peso final }- \text { Peso inicial }}{\text { Peso inicial }} \times 100 \quad$ Ec. 1

Donde:

Peso final es el peso medido después de la incubación, lavado y secado

Peso inicial es el peso medido antes de la incubación.

\section{Análisis de datos}

Se utilizó un diseño completamente al azar en arreglo factorial $6 \times 2 \times 2 \times 2$ cuyos factores fueron seis niveles de urea, dos dietas, denominada variable D1: dieta de alta actividad ruminal (AAR) y dieta de baja actividad ruminal (BAR), dos tamaños de partícula, denominada variable D2: picado a 2 $\mathrm{cm}$ y molido a $2 \mathrm{~mm}$, y dos tiempos de incubación en rumen, denominada variable D3: 48 y 96 horas. Como resultado, se obtuvieron 48 tratamientos con cuatro repeticiones, dando un total de 192 unidades experimentales.

Los datos se analizaron mediante regresión lineal múltiple por pasos sucesivos (Stepwise Regression) que permitió seleccionar las variables que mejor predicen la degradabilidad de MS de entre todas las que se incluyeron en el estudio (Rojo, 2007). Las variables cualitativas D1 (AAR y BAR), D2 (2 cm y $2 \mathrm{~mm}$ ) y D3 (48 y 96 horas) fueron reemplazadas por coeficientes Dummy 1 o -1. Así, a la Dieta AAR, tamaño $2 \mathrm{~cm}$ y 48 horas de incubación les correspondió el valor de 1 y a la dieta BAR, tamaño $2 \mathrm{~mm}$ y 96 horas de incubación, les correspondió el valor de -1. Esas variables Dummy permitieron incluir los factores cualitativos en la 
regresión múltiple y así determinar el efecto de cada uno de estos sobre la variable de respuesta, o descartar en ausencia de efecto (Rawlings et al., 1998). Mediante el reemplazo en la ecuación de regresión los valores de los coeficientes Dummy 1 o -1, según la variable de interés, se cuantificó el efecto que los factores estudiados acusaron en conjunto sobre la DMS. Al reemplazar el valor Dummy de una sola variable a la vez y, calculando la diferencia numérica en esa variable en la Ecuación, se determinó el efecto que ese factor ejerció sobre la DMS independientemente de los demás factores estudiados. Se utilizó el software estadístico SPSS 22 (IBM, 2013).

\section{Resultados y discusión}

$\mathrm{L}$ as variables $\mathrm{N}$ Nivel de urea, D1 Actividad ruminal y D3 Tiempo en rumen, acusaron un efecto altamente significativo $(\mathrm{P}>0.01)$ sobre la DMS del rastrojo de arroz. Contrariamente, la variable D2 Tamaño de partícula no reflejó efecto sobre la degradabilidad del rastrojo (Cuadro 2).

El modelo general encontrado que explica estas relaciones fue el siguiente:

$\mathrm{DMS}=48.72+2.14(\mathrm{D} 1)-4.47(\mathrm{D} 3)+0.27(\mathrm{~N}) \quad$ Ec. 2

Los resultados muestran que el incremento de urea al sustrato, resultó en un mayor porcentaje de digestibilidad del rastrojo de arroz. Esto es, por cada $\mathrm{g} \mathrm{kg}^{-1}$ de urea añadida, la DMS se incrementó en 0.27 unidades.

De otra parte, la dieta con alta actividad ruminal causó un aumento de +2.14 unidades en la DMS del rastrojo de arroz, o en sentido contrario, una dieta con baja actividad ruminal, se reflejó en una disminución de -2.14 unidades en la DMS. Estos resultados se atribuyen a las características de la dieta de alta actividad ruminal basada en heno de $M$. sativa ajustada a un consumo de mantenimiento y complementada con heno de una mezcla forrajera de baja calidad ofrecida ad libitum + concentrado comercial, que habría causado una lenta tasa de pasaje y consecuentemente una lenta degradabilidad ruminal de la materia seca, respecto de la

Cuadro 2. Coeficientes del modelo de regresión lineal múltiple para la variable Degradabilidad Ruminal de la Materia Seca (DMS) del rastrojo de arroz

\begin{tabular}{cccc}
\hline Variable & Coeficiente & $\begin{array}{c}\text { Error } \\
\text { estándar }\end{array}$ & Significancia [P] \\
\hline Intercepto & 48.72 & 0.26 & $>0.01$ \\
$\mathrm{~N}$ & 0.27 & 0.01 & $>0.01$ \\
$\mathrm{D}_{1}$ & 2.14 & 0.17 & $>0.01$ \\
$\mathrm{D}_{3}$ & -4.47 & 0.17 & $>0.01$ \\
\hline
\end{tabular}

dieta de baja actividad ruminal basada únicamente del heno de la mezcla forrajera y concentrado. Al respecto, ciertos estudios señalan que la dieta puede tener un efecto en la tasa de degradación del material que es incubado; así, en animales que son alimentados con dietas altas en concentrados, la actividad de microorganismos que degradan la celulosa se reduce considerablemente (Ørskov, 2000; Villalobos et al., 2000).

La variable D2 Tamaño de partícula no afectó la respuesta animal, a pesar de que varios autores señalan que aquellas dietas concentradas constituidas por partículas pequeñas se caracterizan generalmente por ser de rápida tasa de pasaje y degradación, respecto de aquellas dietas más groseras que caracterizan a los forrajes las cuales presentan mayor retención y lenta degradación (Rosero y Posada, 2007; Ørskov, 2000), cuyas diferencias explican una correlación positiva entre digestión y tasa de pasaje. Contrariamente, otros autores no reportan efecto alguno del tamaño de partícula sobre la digestibilidad de rastrojos de maíz (Jiménez et al., 2010).

En relación con el tiempo de incubación (D3), se ha demostrado que el tiempo de residencia de los alimentos en el rumen es generalmente de 48 horas (Ørskov y Shands, 1997). No obstante, como lo demuestra la Ecuación 2 y lo confirman otros estudios previos, como el de Nakashima y Ørskov (1990), el coeficiente en la variable D3 arrojó un valor de -4.47 , que corresponde a la reducción proporcional de la DMS del rastrojo de arroz a 48 horas, versus +4.47 de aumento de la DMS cuando es incubada a 96 horas. Esos resultados demuestran un incremento de la digestibilidad de nueve puntos porcentuales al permanecer el sustrato el doble del tiempo en incubación en rumen. Aunque la información sobre la cinética de la degradabilidad de los recursos forrajeros, incluyendo los subproductos agroindustriales como el rastrojo de arroz, es bastante escasa y en algunos casos inexistente en la literatura científica (Naranjo y Cuartas, 2011), varios autores muestran resultados diferentes en el efecto del tiempo de incubación sobre la digestibilidad real (Arredondo et al., 1995; Gojon et al., 1998; Pulido y Leaver, 2000; Salcedo, 2000; Boshini, 2001; Razz et al., 2004; Correa, 2006; Vergara y Araujo, 2006; Rossi et al., 2008; Naranjo y Cuartas, 2011; Barrera et al., 2015) debido a múltiples factores que afectan los resultados, entre estos se pueden contar el modelo matemático para determinar los parámetros de cinética ruminal, las fuentes de variación asociadas a las técnicas y otros factores relacionados al material utilizado (Naranjo y Cuartas, 2011; Barrera et al., 2015).

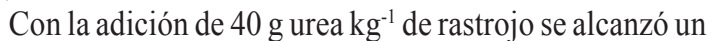
incremento de la degradabilidad ruminal hasta de 11 puntos porcentuales (Figura 1). El aumento de la DMS con la adición de urea concuerda con estudios anteriores que afirman que el tratamiento con urea incrementa la digestibilidad del rastrojo de arroz (Yulistiani y Gallagher, 2000; Wanapat et al., 


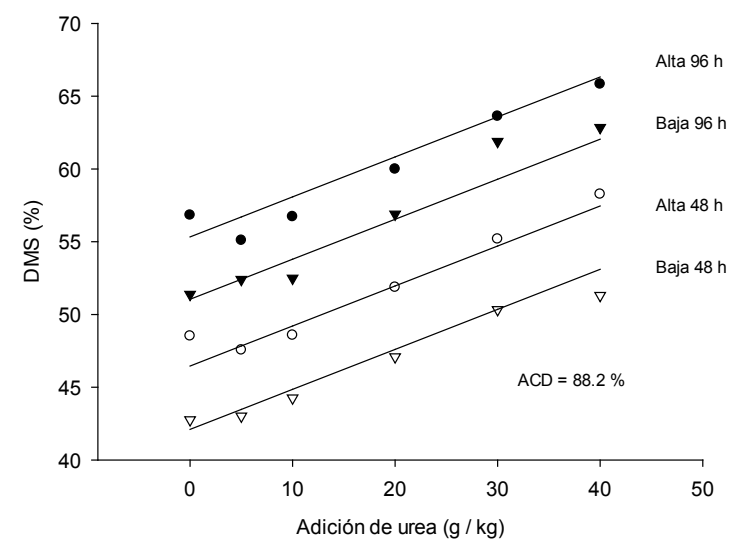

Figura 1. Degradabilidad ruminal de la materia seca con distintos niveles de adición de urea, a diferentes tiempos de incubación en rumen y dietas de alta y baja actividad ruminal.

2009), y también en otros subproductos como el maíz donde la amonificación causó incrementos en la digestibilidad de nutrientes (Jiménez et al., 2010). Adicionalmente, estudios de digestibilidad por el método in situ, con una adición de 20 a $50 \mathrm{~g}$ urea $\mathrm{kg}^{-1}$ rastrojo presentaron un incremento de la DMS de alrededor de un 15\% adicional (Van Soest, 2006). Por su parte, Vadiveloo y Fadel (2009) utilizando el método in vitro encontraron que el aumento de la digestibilidad asociado al uso de urea, fue más efectivo cuando se aplicó sobre la planta completa que únicamente en las fracciones botánicas. De hecho, múltiples estudios sobre el uso de urea en rastrojos de cereales como el arroz y trigo, han demostrado mejoras en los niveles de nitrógeno en la dieta de los rumiantes (Fahmy y Orskov, 1984) lo cual estaría asociado a la síntesis de proteína microbiana y a una disminución del poder ligante de la lignina a la hemicelulosa y celulosa, dado que el $\mathrm{N}$ amoniacal tiene efecto alcalino deslignificante (Klopfenstein, 1978), permitiendo un incremento de la digestibilidad (Horton y Steacy, 1979, y por lo tanto, el consumo (Badurdeen et al., 1994).

\section{Conclusiones}

E n las condiciones en que se desarrolló este estudio, la adición de urea al rastrojo de arroz en un ambiente con dietas de alta actividad ruminal, fueron capaces de aumentar la degradabilidad ruminal de la materia seca del sustrato a mayor tiempo de incubación en rumen, en tanto, el tamaño de partícula aparentemente no fue un criterio relevante. Por lo expuesto, se podría considerar al rastrojo de arroz tratado con urea, como una fuente potencial para ser incluida parcialmente en la dieta de rumiantes.

\section{Agradecimientos}

Al Dr. Brian Palmer, Asesor Ministro de Agricultura Ganadería, Acuacultura y Pesca, por el diseño y dirección de la presente investigación. Al equipo técnico del Centro Nacional de Producción y Mejoramiento Genético El Rosario de la Subsecretaría de Ganadería (ex MAGAP); a la Asociación Junta General de Usuarios de Sistema de Riego y Drenaje "América Lomas"; a la Facultad de Medicina Veterinaria y Zootecnia de la Universidad Central del Ecuador y a la Estación experimental Santa Catalina del INIAP.

\section{Referencia bibligráfica}

Arreaza, LC., Sánchez, DE., Abadía, B. 2005. Degradabilidad ruminal de fracciones de carbohidratos en forrajes tropicales determinada por métodos in vitro e in situ. Revista Corpoica, 6(1): 57-62.

Arredondo, SS., Jahn, BE., Ovalle, MC. 1995. Degradabilidad ruminal de distintos componentes de la planta de Tagabaste (Chamaecytisus proliferus subsp. Palmensis) mediante el uso de la técnica de novillos fistulados en el rumen. Agricultura Técnica (Chile), 57: 127-135.

Badurdeen, AL., Ibrahim, MNM., Schiere, JB. 1994. Methods to improve utilization of rice straw. II. Effects of different levels of feeding on intake and digestibility of untreated and urea ammonia treated rice straw. AsianAustralian Journal Animal Science, 7: 165-169.

Barrera, AE., Avellaneda, JH., Tapia, EO., Peña, MM., Molina, CA., Casanova, LM. 2015. Composición química y degradación de cuatro especies de Pennisetum sp. Ciencia y Tecnología, 8(2): 13-27.

Bartaburu, D., Montes, DE., Pereira, M. 2008. Utilización de la paja de arroz en la alimentación animal. ln: INIA, 
Ministerio de Ganadería, Agricultura y Pesca (MGAP), Instituto Plan Agropecuario. Alternativas tecnológicas para enfrentar situaciones de crisis forrajeras. Montevideo, Uruguay.

Boshini, C. 2001. Degradabilidad in situ de la materia seca, proteína y fibra del forraje de morera (Morus alba). Agronomía Mesoamericana, 12(1): 79-87.

Correa, HJ. 2006. Cinética de la liberación ruminal de macrominerales en pasto kikuyo (Pennisetum clandestinum) cosechado a dos edades de rebrote. Universidad Nacional de Colombia. Sede Medellín.

Drake, D., Nader, G., Forero, L. 2002. Feeding Rice Straw to Cattle. Publication 8079. University of California, Division of Agricultural and Natural Resources.

Fahmy, ST., Orskov, ER. 1984. Digestion and utilization of straw. Effect of different supplements on the digestion of ammonia treated straw. Animal Production, 88: 75-81.

FASS. 2010. Guide for the Care and Use of Agricultural Animals in Research and Teaching. Federation of Animal Science Societies, Third Edition, January 2010.

France, J., Theodorou, MK., Lowman, RS., Beever, DE. 2000. Feed evaluation for Animal Production. In MK. Theodorou, J. France (eds) "Feeding systems and feed evaluation models". CAB International.

Garg, MR. 2012. Balances feeding for improving livestock productivity: increase in milk roduction and nutrient use efficiency and decrease in methane emission. FAO Animal Production, Rome, Italy, Rep.

Giraldo, LA., Gutiérrez, LA., Rúa, C. 2007. Comparación de dos técnicas: in vitro e in situ para estimar la digestibilidad verdadera en varios forrajes tropicales. Revista Colombiana de Ciencias Pecuarias, 2007(20): 269-279.

Gunun, P., Wanapat, M., Anantasook, N. 2013. Effects of physical form and urea treatment of rice straw on rumen fermentation, microbial protein synthesis and nutrient digestibility in dairy steers. Asian-Australian Journal Animal Science, 26(12): 1689-1697.

Haryanto, B. 2014. Maximizing Utilization of Energy from Crop By-products. WARTAZOA Indon. Bull. Animal Veterinary Science, 24(1): 30-39.

Horton, G.M.J., Steacy, M. 1979. Effect of anhidrous ammonia treatment of the intake and digestibility of cereal straw by steers. Journal Animal Science, 48: 1239-1249.

IBM Corp. 2013. IBM SPSS Statistics for Windows, Version 22.0. Armonk, NY: IBM Corp.

INEC-ESPAC. 2014. Encuesta de superficie y producción agrícola continua ESPAC 2014. Dirección de Estadísticas Agropecuarias y Ambientales, INEC.

INTA. 2014. Nutrición animal aplicada. INTA-EEA Balcarce, mayo de 2014.

Jackson, M.G. 1997. Rice straw as livestock feed. World Animal Review, 1977(23): 34-40.

Jiménez, RA., San Martín, FH., Huamán, HU., Ara, MG.,
Arbaiza, TF., Guamán, AC. 2010. Efectos del tamaño de partícula y tipo de amonificación-conservación sobre la digestibilidad y consumo del rastrojo de maíz en ovinos. Revista de Investigaciones Veterinarias del Perú, 21(1): 19-25.

Klopfenstein, T. 1978. Chemical treatment of crop residues. Journal Animal Science, 46: 841-848.

Liu, JX., Orskov, ER., Chen, XB. 1999. Optimization of steam treatment as a method for upgrading rice straw as feeds. Animal Feed Science and Technology, 76(3): 345-357.

Malik, K., Tokkas, J., Anand, RC. Kumari, N. 2015. Pretreated rice straw as an improved fodder for ruminants-An overview. Journal Applied and Natural Science, 7(1): $514-520$.

Manterola, H., Cerda, D. 2017. Uso de residuos agroindustriales en alimentación de rumiantes y métodos para mejorar su eficiencia de uso. Sitio Argentino de Producción animal.

Moran, J. 2005. Tropical Dairy Farming: Feeding Management for Small Holder Dairy Farmers in the Humid Tropics. 1a. Edition, Victoria, Australia, Landlinks Press.

Nakashima, Y., Ørskov, ER. 1990. Rumen degradation of straw 9. Effect of cellulase and ammonia treatment on different varieties of rice straws and their botanical fractions. Animal Production, 50(2): 309-317.

Naranjo, JF., Cuartas, CA. 2011. Caracterización nutricional y de la cinética de degradación ruminal de algunos de los recursos forrajeros con potencial para suplementación de rumiantes en el trópico alto de Colombia. CES Medicina Veterinaria y Zootecnia, 6(1): 9-19.

Ørskov, ER., Shand, WJ. 1997. Use of the nylon bag technique for protein and energy evaluation and for rumen environment studies in ruminants. Livestock Research for Rural Development. 9(3). Retrieved August 28, 2017, from http://www.lrrd.org/lrrd9/1/orskov.htm

Ørskov, ER. 2000. The in situ technique for the estimation of forage degradability in ruminants. In: D.L. Givens, E. Owen, RFE. Axford, HA. Omed (eds.) "Forrage evaluation in Ruminant Nutrition, CAB International.

Pulido, R., Leaver, JD. 2000. Degradabilidad ruminal del forraje disponible en la pradera y del aparentemente consumido por vacas lecheras. Pesquisa Agropecuaria Brasileira, 35(5): 1003-1009.

Pond, WG., Church, DC., Pond, KR. 2006. Fundamentos de Nutrición y Alimentación de Animales. 2a ed. México: Limusa SA.

Posada, SL., Noguera, RR. 2005. Técnica in vitro de producción de gases: Una herramienta para la evaluación de alimentos para rumiantes. Livestock Research for Rural Development, 17(4): 12-19.

Rawlings, JO., Pentula, SG., Dickey, DA. 1998. Applied regression analysis: a research tool, 2a Edition, NYUSA, Editorial Springer. 
Razz, R., Clavero, T., Vergara, J. 2004. Cinética de degradación in situ de la Leucaena leucocephala y Panicum maximum. Revista Científica Universidad de Zulia (Venezuela), 16(005): 424-430.

Rojo, JM. 2007. Regresión Lineal Múltiple. Instituto de Economía y Geografía, Madrid, España.

Rosero, R. y Posada, SL. 2007. Modelación de la cinética de degradación de alimentos para rumiantes. Revista Colombiana de Ciencias Pecuarias, 20: 174-82.

Rossi, CA., González, GL., Magistris, AA 2008. Degradabilidad in situ de forrajeras del pastizal natural en un sistema silvopastoril con salicáceas. Zootecnia tropical, 26(3): 257-259.

Saddul, D., Jelan, ZA., Liang, JB., Halim, RA. 2005. Evaluation of mulberry (Morus alba) as potential feed supplement for ruminants: The effect of plant maturity on in situ disappearance and in vitro intestinal digestibility of plant fractions. Asian-Australian Journal Animal Science, 18(11): 1569-1574.

Salcedo, G. 2000. Degradabilidad ruminal de la hierba en praderas aprovechadas bajo pastoreo rotacional, en la zona costera de Cantabria. Investigación Agropecuaria: Producción, Sanidad Animal, 15(3): 125-135.

Sarnklong, C., Cone, JW., Pellikaan, W., Hendriks, WH. 2010. Utilization of rice straw and different treatments to improve its feed value for ruminants: A review. AsianAustralian Journal Animal Science, 23(5): 680-692.

Tobal, CF. 1999. Evaluación de los alimentos a través de los diferentes métodos de digestibilidad. Revista Colombiana de Ciencias Pecuarias, 1: 520-525.

Torres, GG., Arbaiza, T., Carcelén, CF. Lucas, AO. 2009. Comparación de las técnicas in situ, in vitro y enzimática (Celulasa) para estimar la digestibilidad de forrajes en ovinos", Revista de Investigación Veterinaria, 20(1): 5-9.

Vadiveloo, J., Fadel, JG. 2009. The response of rice straw varieties to urea treatment. Animal Feed Science and
Technology, 151(3): 291-298.

Van Soest, PJ. 2006. Rice straw, the role of silica and treatments to improve quality. Animal Feed Science and Technology, 130(3): 137-171.

Vergara, LJ., Araujo, O. 2006. Producción, composición química y degradabilidad ruminal in situ de Brachiaria humidicola (Rendle) Schweick en el bosque seco tropical. Faculta de Ciencias Veterinarias-LUZ 16(3): 236-248.

Villalobos, GC., González, VE., Ortega, SJ. 2000. Técnicas para estimar la degradación de proteína y materia orgánica en el rumen y su importancia en rumiantes en pastoreo. Técnica Pecuaria, 38(002): 119-134.

Wanapat, M., Polyorach, S., Boonnop, K., Mapato, C., Cherdthong, A. 2009. Effects of treating rice straw with urea or urea and calcium hydroxide upon intake, digestibility, rumen fermentation and milk yield of dairy cows. Livestock Science, 125(2): 238-243.

Yulistiani, D., Gallagher, JR. 2000. Nutritive value improvement of rice straw varieties for ruminants as ditermined by chemical composition and in vitro organic matter digestibility. Ilmu. Ternak. Dan. Veterinary, 5(1): 23-31.

Yulistiani, D., Jelan, ZA., Lian, JB. 2008. Degradability of Mulberry (Morus alba) and rice bran in the rumen of Sheep Fed of different diets. Indonesian Journal Animal Veterinary Science, 13(4): 264-272.

Yulistiani, D., Jelan, ZA., Lian, JB., Yaakub, H., Abdullah, N. 2015. Effects of suplementation of Mulberry (Morus alba) foliace anda urea-rice brand as fermentable energy and protein source in sheep fed urea-treated rice straw based diet. Asian-Australian Journal Animal Science, 28(4): 494-501. 